\title{
The Effect of a Decision-Support mHealth Application on Maternal and Neonatal Outcomes in Two District Hospitals in Rwanda: Quasi-Experimental Study
}

\author{
Aurore Nishimwe ( $\nabla$ naurore@cartafrica.org )

\section{Latifat Ibisomi} \\ University of the Witwatersrand Faculty of Health Sciences \\ Marc Nyssen \\ Vrije Universiteit Brussel

\section{Daphney Nozizwe Conco} \\ University of the Witwatersrand Faculty of Health Sciences
}

University of the Witwatersrand Faculty of Health Sciences https://orcid.org/0000-0002-8019-2561

\section{Research}

Keywords: Maternal Outcome, Neonatal Outcome, BEmONC, mHealth, Safe Delivery Application, Rwanda

Posted Date: September 23rd, 2020

DOI: https://doi.org/10.21203/rs.3.rs-79352/v1

License: (c) (i) This work is licensed under a Creative Commons Attribution 4.0 International License. Read Full License 


\section{Abstract}

Background: Globally, mobile health (mHealth) applications are known for their potential to improve healthcare providers' access to relevant and reliable health information. Besides, electronic decision support tools, such as the Safe Delivery mHealth Application (SDA), help to reduce clinical errors and to ensure quality care at the point of service delivery. The current study assessed the effects of the SDA on basic emergency obstetric and newborn care (BEmONC) outcomes for the most frequent complications in Rwanda; post-partum haemorrhage (PPH) and newborn asphyxia and its complications.

Methods: The study adopted a quasi-experimental design. A pre-intervention record review of the BEmONC outcomes: Apgar score and PPH progressions, was conducted. The intervention took place in two district hospitals in Rwanda and entails the implementation of the SDA for six months. Six months' post-intervention, the effect of the SDA on BEmONC outcomes was evaluated. The study included 327 participants (114 cases of PPH and 213 cases of neonatal complications). The analysis compared the outcome variables between the baseline and the endline data. Fisher's exact test was used to compare the proportions and test between-group differences and significance level set at $p<0.05$.

Results: Unstable newborn outcome following neonatal resuscitation was recorded in $61.90 \%$ and $27.59 \%$ newborns cases at baseline and endline respectively, P-value $=0.000$. Unstable maternal outcome following PPH management was recorded in $19.40 \%$ and $6.38 \%$ maternal cases at baseline and endline respectively, P-value $=0.048$. There was a significant association between the SDA intervention and newborns' and maternal' outcomes following neonatal resuscitation and PPH management, 6 months after baseline.

Conclusion: The use of the SDA supported nurses and midwives in the management of PPH and neonatal resuscitation thus improved maternal and neonatal outcomes after 6 months of the SDA intervention.

\section{Plain English Summary}

Globally, mobile health (mHealth) applications are known for their potential to improve healthcare providers' access to relevant and reliable health information. Besides, electronic decision support tools, such as the Safe Delivery mHealth Application (SDA), help to reduce clinical errors and to ensure quality care at the point of service delivery. The current study assessed the effects of the SDA on basic emergency obstetric and newborn care (BEmONC) outcomes for the most frequent complications in Rwanda; post-partum haemorrhage (PPH) and newborn asphyxia and its complications.

This study adopted a quasi-experimental design. A pre-intervention record review of the BEmONC outcomes: Apgar score and PPH progressions, was conducted. The intervention took place in two district hospitals in Rwanda and entails the implementation of the SDA for six months. Six months' post-intervention, the effect of the SDA on BEmONC outcomes was evaluated. The study included 114 cases of PPH and 213 cases of neonatal complications. The analysis compared the outcome variables between the baseline and the endline data.

Unstable newborn outcome following neonatal resuscitation was recorded in $61.90 \%$ and $27.59 \%$ newborns cases at baseline and endline respectively. Unstable maternal outcome following PPH management was recorded in $19.40 \%$ and $6.38 \%$ maternal cases at baseline and endline respectively. There was a significant association between the SDA intervention and newborns' and maternal' outcomes, 6 months after baseline. 
The use of the SDA supported nurses and midwives in the management of PPH and neonatal resuscitation thus improved maternal and neonatal outcomes after 6 months of the SDA intervention.

\section{Background}

Mhealth refers to the practice of medicine and public health, supported by mobile devices (mobile phones, smartphones, tablets, smartwatches) [1]. Evidence suggests that mHealth applications are innovative approaches to improve the delivery of health care services [2]. Further, mhealth tools can help to reduce clinical errors and ensure quality care at the point of service delivery. There is growing evidence of the effectiveness of mHealth interventions in maternal and newborn health programs in low and middle-income countries (LMICs), particularly to improve adherence to treatment, meeting appointments, facilitate data collection, and the development of supportive networks for health professionals[3-6].

Among the many interventions currently being implemented to support maternal and newborn healthcare services, mHealth applications have been widely used in low-resources settings $[4,7-9]$ as a potential solution to maximize health providers' efficiency, health outcomes[9, 10,11] and improve service utilization[14]. Common areas of application of mHealth tools include point-of-care decision-making support; provider-to-provider communication; and data collection[15, 16]. Though mHealth interventions are well received by healthcare providers $[6,17,18]$ information about their effectiveness with regards to patient outcomes is limited[5, 9, 19].

Many interventions on the use of mHealth for improving maternal and newborns' health outcomes in sub-Saharan Africa mainly focused on timely access to health facilities including reminders for antenatal appointments and referrals of mothers[20]. Few mHealth applications are addressing quality service delivery with the main focus on clinical decision support in order to improve maternal and newborns outcomes[21]. The Safe Delivery mHealth Application (SDA) is one of the recent mHealth applications (loaded in smartphones) which avails BEmONC clinical guidelines to support nurses and midwives' clinical decisions[22].

Rwanda, a sub-Saharan African country, reports high maternal and neonatal deaths. In 2015, Rwanda's maternal mortality was estimated at 290 deaths per 100000 live births and its neonatal mortality rate was 20 deaths per 1000 live births, respectively[23] despite $89,8 \%$ of deliveries taking place in healthcare facilities attended by health professionals [20,21]. Common causes of these deaths are preventable including postpartum hemorrhage (71.6\%) and newborn asphyxia and its complications (36.7\%)[26]. Challenges faced by healthcare providers include poor access to clinical guidelines and the lack of timely response to pregnancy complications during delivery care[27]. It is in this context that a mHealth application - a clinical decision support tool (SDA) to facilitate easy access to maternal and neonatal guidelines for routine and emergency obstetric and neonatal care in Rwanda was introduced. This study assessed the effects of the SDA on basic emergency obstetric and newborn care (BEmONC) outcomes for the most frequent delivery-related complications of postpartum haemorrhage (PPH) and newborn asphyxia in Rwanda.

\section{Methods}

\section{Study design}

This was a quasi-experimental study done over a 14-month period. The study was conducted in three phases. The first phase was the pre-SDA intervention baseline study over a period of six months (February 2019 - July 2019). During this phase, a cross-sectional study was conducted, using a record review, to document maternal and 
neonatal outcomes; PPH and Apgar score progressions. The second phase, the SDA intervention (August 2019 September 2019) comprised of the capacity building of nurses and midwives on the usage of the SDA; the SDA provision; the SDA piloting, and the launch of the SDA which marked the start of the SDA implementation. The last phase was the six months of post-SDA intervention (October 2019- March 2020). Phase three encompassed the implementation of the SDA and a cross-sectional study, using a record review to document maternal and neonatal outcomes; PPH and Apgar score progressions at endline.

\section{Study Setting}

The study was conducted in two district hospitals in Rwanda: Masaka hospital in Kigali, an urban province; and Nyamata hospital located in the eastern rural province[25]. The two hospitals were selected out of 12 district hospitals in the two provinces because both had a high number of deliveries per year[23]. Compared to other ruralbased district hospitals, Nyamata reported the highest maternal and neonatal mortality rates[27].

\section{Study Participants}

The study participants included nurses and midwives who used the SDA in both Masaka and Nyamata district hospitals. The inclusion criteria for nurses and midwives were set as follows: having a work experience of at least 6 months in obstetric care and willing to participate in the study. The study sample also included records on neonatal complication cases and PPH cases. The inclusion criteria for the cases of neonatal complications were set as babies born with asphyxia (Apgar score $\leq 7$ ) and were subject to neonatal resuscitation. We excluded preterm births ( $\leq 36$ completed weeks of gestation) and births with major congenital malformations because prematurity and congenital complications other than the newborn asphyxia could influence the newborn outcome after resuscitation[28]. We have also excluded records with missing data on Apgar scores and neonatal outcome. On the other hand, the inclusion criteria for PPH cases were set as women who had a recorded amount of blood loss more than or equal to $500 \mathrm{mls}$ and were subjected to PPH management. The records with missing data on PPH progression and maternal outcomes were also excluded. The study was conducted in three phases. Phase one, preintervention, included 126 cases of newborns asphyxia and 67 cases of PPH. Phase two, intervention, involved 33 midwives and 21 nurses from both Masaka and Nyamata district hospitals. Phase three, post-intervention, comprised 87 cases of newborns asphyxia and 47 cases of PPH. The flowchart in Fig. 1 shows the number of neonatal complication cases and PPH cases considered in the baseline group (before the introduction of SDA) and the endline group (after SDA).

\section{Description of the intervention: Implementation of the Safe Delivery mhealth Application (SDA)}

The SDA is a smartphone application developed by the Maternity Foundation, Copenhagen University, and the University of Southern Denmark. It is designed to support nurses and midwives in clinical decision making, by providing them with instant access to evidence-based BEmONC clinical guidelines and a selection of essential preventive protocols in a user-friendly format. The content of the SDA is primarily based on WHO clinical BEmONC guidelines and has been validated by an international group of global health experts[29]. The SDA contains easy to understand animated instruction videos, action cards, essential drugs lists with their indications, practical procedures guidelines, and a learning platform[30]. All features and functions in the SDA are designed for lowliteracy and low-income settings and work offline once downloaded. It can thus, be adapted to local contexts and policies. The SDA can be downloaded free of charge for iPhone at https://itunes.apple.com/dk/app/safe- 
delivery/id985603707?mt=8 and for Android at https://play.google.com/store/apps/details?

$\mathrm{id}=\mathrm{dk} \cdot$ maternity. safedelivery\&hl=en.

Each of the study hospitals (Masaka and Nyamata) was allocated three smartphones, with pre-installed English and French versions of the SDA. The nurses and midwives in both hospitals received an explanation of the nature and purpose of the research and half-day training session on the use of the SDA. The majority of participants have downloaded the SDA on their personal smartphones during the intervention. The training session encompassed: an introduction to the research project; an overview of the SDA development and global outreach; description of the SDA features and modules; and hands-on practice on using the SDA as a job-aid and as a learning tool. This was followed by the six months' intervention period, during which nurses and midwives provided BEmONC services with the assistance of the SDA installed on their personal smartphones or the smartphones provided by the researcher. During this period, the provided six smartphones were made available to the team on duty at all times for use as a backup in case the participants experienced problems with their own smartphones. Nurses and midwives were expected to use the SDA as often as they wished. The intervention also entailed two visits per week by two research assistants to each of the study hospitals. During the visits, the research assistants monitored the SDA use by nurses and midwives.

\section{Data collection}

Data extraction forms were used to retrieve information on all cases of PPH and neonatal complications recorded in delivery registers. These were extracted and reviewed six months' pre-SDA intervention and six months' post-SDA intervention. The delivery registers contain information on obstetric and neonatal care including the Apgar scores and PPH progressions following neonatal resuscitation and PPH management. Newborn deaths or admission to the neonatal intensive care unit (NICU) or to neonatologist's service due to an unstable outcome (Apgar score $<7$ ) after neonatal resuscitation was used as the primary outcome measure to determine the failure of neonatal resuscitation. The mothers' referral to a higher-level facility (referral hospital) following an unstable outcome (persistent bleeding) after PPH management was considered as the primary outcome to measure the failure of PPH management at the district hospital. On the newborn side, we obtained data on Apgar scores progression and neonatal outcome following 10 minutes' neonatal resuscitation before and after the use of the SDA. While on the mother's side, we obtained data on maternal outcomes following PPH management before and after the use of the SDA.

\section{Ethics approval}

This study has been approved by the Human Research Ethics Committee of the University of the Witwatersrand (M190258) and the University of Rwanda, College of Medicine and Health Sciences' Institutional Review Board (No.377/CMHS IRB/2018). Permission has been granted by the hospitals.

\section{Statistical analysis}

The data were checked for errors and exported from Microsoft Excel (Microsoft Corporation) to Stata version 16 (StataCorp LLC) for cleaning and analysis. Descriptive summary statistics were computed on data including the hospital, age of the mother at childbirth, the weight of the newborn at birth, sex of the newborn, mode of delivery, the leading clinician during delivery, Apgar scores at 1,5 and 10 minutes and the resuscitation action taken for the newborns. For the maternal outcome, descriptive statistics included the hospital, age of the mother at delivery, gestational age in weeks, Mode of delivery, the leading clinician during delivery, Blood loss in milliliters, causes of $\mathrm{PPH}$, the resuscitation action taken, mother outcome and cause of maternal death if dead. Further, the neonatal outcomes and maternal outcomes were compared at baseline and endline using Fisher's exact test. significance level was set at $p<0.05$. 


\section{Results}

\section{Background Characteristics \\ Cases of newborns asphyxia}

The analysis included 126 cases of newborns asphyxia in the baseline and 87 in endline groups over the study period. Table 1 shows that the majority of cases were from Masaka district hospital in the baseline group (54.76\%) while in the endline group more cases were from Nyamata district hospital (56.32\%). There were more male newborns for the baseline group $(n=79,62.70 \%)$ and the endline group $(n=54,62.07 \%)$. The most frequent mode of delivery was the spontaneous vaginal delivery in both the baseline group $(n=77,61.11 \%)$ and the endline groups ( $n$ $=45,51.72 \%)$. The leading clinicians during delivery, also in charge of neonatal resuscitation were mainly midwives $(n=71,56.35 \%)$ in the baseline group and $(n=44,50.57 \%)$ in the endline group. The average age of the mother was similar, 28 years and 27 years in the baseline and endline groups, respectively. The average weight of the newborn was similar, 2900 grams, and 2800 grams in the baseline and endline groups, respectively. The average Apgar score at birth was the same, 6/10 in both the baseline and endline groups. However, the average Apgar score after 10 minutes of resuscitation was lower in the baseline group (8/10) than the endline group (9/10). The resuscitation actions taken were mostly dominated by aspiration and ventilation with Ambu bag in both groups, baseline (81.74\%), and endline (79.30\%). The endline group had higher numbers of newborns with stable outcome after resuscitation ( $n=64,73.56 \%)$ than the baseline group $(n=40,31.75 \%)$. Overall, the endline group had fewer newborns transferred to NICU or neonatology service $(n=24,27.59 \%)$ than the baseline group $(n=78,61.90 \%)$. 
Table 1

Characteristics of study Participants_Cases of newborns asphyxia $(\mathrm{N}=213)$

\begin{tabular}{|c|c|c|}
\hline & Baseline n (\%) & Endline n (\%) \\
\hline \multicolumn{3}{|l|}{ Hospital Affiliation } \\
\hline Masaka District Hospital & $69(54.76)$ & $38(43.68)$ \\
\hline Nyamata District Hospital & $57(45.24)$ & $49(56.32)$ \\
\hline \multicolumn{3}{|l|}{ Gender } \\
\hline Male & $79(62.70)$ & $54(62.07)$ \\
\hline Female & $47(37.30)$ & $38(37.97)$ \\
\hline \multicolumn{3}{|l|}{ Mode of Delivery } \\
\hline Caesarean Section & 49 (38.89) & $42(48.28)$ \\
\hline Spontaneous Vaginal Delivery & $77(61.11)$ & $45(51.72)$ \\
\hline \multicolumn{3}{|l|}{ Leading Clinician of the Delivery } \\
\hline Doctor & 49 (38.89) & $42(48.28)$ \\
\hline Midwife & $71(56.35)$ & $44(50.57)$ \\
\hline Nurse & $6(4.76)$ & $1(1.15)$ \\
\hline \multicolumn{3}{|l|}{ Resuscitation Actions } \\
\hline Aspiration, Ventilation with Ambu bag & $103(81.74)$ & $69(79.30)$ \\
\hline Aspiration, Ventilation, Oxgenotherapy & $12(9.52)$ & $6(6.90)$ \\
\hline CPR, Ventilation, Oxygenation & $11(8.73)$ & $12(13.80)$ \\
\hline \multicolumn{3}{|l|}{ Outcome after 10 minutes Resuscitation } \\
\hline Transferred to NICU or Neonatology Service & $78(61.90)$ & $24(27.59)$ \\
\hline Skin to skin bonding with mother & 39 (30.95) & $63(72.41)$ \\
\hline Dead & $9(7.14)$ & $0(0)$ \\
\hline Weight of the newborn, grams, Median (IQR) & $2900(880)$ & $2800(800)$ \\
\hline Mother's age, years, Median (IQR) & $28(7)$ & $27(7)$ \\
\hline \multicolumn{3}{|l|}{ Apgar Scores, Median (IQR) } \\
\hline At 1 minute & $6(2)$ & $6(2)$ \\
\hline At 5 minutes & $7(2)$ & $7(1)$ \\
\hline At 10 minutes & $8(2)$ & $9(2)$ \\
\hline
\end{tabular}




\section{Cases of Postpartum haemorrhage (PPH)}

The analysis included 114 cases of Post-partum haemorrhage(PPH): 67 in baseline and 47 in endline groups for both Masaka and Nyamata district hospitals over the study period. Table 2 shows that the majority of cases were from Masaka district hospital in the baseline group (53.73\%) while in the endline group more cases were from Nyamata district hospital $(55.32 \%)$. The most frequent mode of delivery was the spontaneous vaginal delivery in both the baseline group $(n=47,70.15 \%)$ and the endline groups $(n=34,72.34 \%)$. The leading clinicians of the delivery, also in charge of PPH management were mainly midwives $(n=36,53.73 \%)$ in the baseline group and $(n=$ $25,53.19 \%)$ in the endline group. The average age of the mother was similar, 28 years in the baseline and endline groups, respectively. The average gestational age was similar, 39 weeks in the baseline and endline groups, respectively. The mean estimated amount of blood loss was $1167.16 \mathrm{mls}$ in the baseline group and $1178.72 \mathrm{mls}$ in the endline group. The most frequent cause of PPH was the uterine atony in both the baseline $(71.64 \%)$ and the endline group (70.21\%). The resuscitation actions taken were mostly dominated by administering oxytocin, IV fluids, and blood transfusion in both groups, baseline (37.31\%), and endline (55.32\%). The endline group had higher numbers of women with stable outcomes after PPH management $(n=44,93.62 \%)$ than the baseline group $(n=55$, 77. $61 \%)$. Overall, the endline group had less women transferred to referral hospitals after PPH management $(n=3.6$, $38 \%)$ than the baseline group $(n=13,19.40 \%)$. 
Table 2

Characteristics of study Participants_Cases of Post - Partum Haemorrhage ( $N=114)$

\begin{tabular}{|c|c|c|}
\hline & Baseline n (\%) & Endline n (\%) \\
\hline \multicolumn{3}{|l|}{ Hospital Affiliation } \\
\hline Masaka District Hospital & $36(53.73)$ & $21(44.68)$ \\
\hline Nyamata District Hospital & $31(46.27)$ & $26(55.32)$ \\
\hline \multicolumn{3}{|l|}{ Mode of Delivery } \\
\hline Caesarean Section & $20(29.85)$ & $13(27.66)$ \\
\hline Spontaneous Vaginal Delivery & $47(70.15)$ & $34(72.34)$ \\
\hline \multicolumn{3}{|l|}{ Leading Clinicians of the Delivery } \\
\hline Doctor & $20(29.85)$ & $13(27.66)$ \\
\hline Midwife & $36(53.73)$ & $25(53.19)$ \\
\hline Nurse & $11(16.42)$ & $9(19.15)$ \\
\hline \multicolumn{3}{|l|}{ Causes of PPH } \\
\hline Uterine atony & $48(71.64)$ & $33(70.21)$ \\
\hline Cervical tear & $11(16.42)$ & $7(14.89)$ \\
\hline Retention of Placenta & $8(11.94)$ & $7(14.89)$ \\
\hline \multicolumn{3}{|l|}{ Resuscitation Actions } \\
\hline Oxytocin, IV fluids & $23(34.33)$ & $7(14.89)$ \\
\hline Oxytocin, IV fluids, Transfusion & $25(37.31)$ & $26(55.32)$ \\
\hline Repair of tear, IV fluids & $11(16.42)$ & $7(14.89)$ \\
\hline Removal of Placenta, IV fluids & $8(11.94)$ & $7(14.89)$ \\
\hline \multicolumn{3}{|c|}{ Maternal outcome after PPH Management } \\
\hline Transferred to Referral Hospital & $13(19.40)$ & $3(6.38)$ \\
\hline Stable & $52(77.61)$ & $44(93.62)$ \\
\hline Dead & $2(2.99)$ & $0(0)$ \\
\hline Mother's age, years, Median(IQR) & $28(5)$ & $28(5)$ \\
\hline Gestational age, weeks, Median(IQR) & $39(0)$ & $39(0)$ \\
\hline Blood loss in Mls, Mean (SD) & $1167.16(276.55)$ & $1178.72(257.02)$ \\
\hline
\end{tabular}




\section{Pre-Post Differences in Newborns outcomes following neonatal resuscitation}

We found a statistically significant association between the SDA intervention and newborns' outcomes following neonatal resuscitation, 6 months after baseline (Table 3). Among 213 cases of newborns complications who were included in the study, stable outcome following neonatal resuscitation was recorded in $30.95 \%$ and $72.41 \%$ at baseline and endline respectively. Transferred (unstable) newborns were $61.90 \%$ and $27.59 \%$ at baseline and endline respectively. While death following neonatal resuscitation appeared in $7.14 \%$ for the baseline group only. No death was recorded in the endline group. There were significant differences in the proportions values of the three categories of newborns' outcomes (stable, transferred, and dead) following neonatal resuscitation at baseline, and at endline, the Fisher's exact test p-value was 0.000 .

Table 3

Newborns Outcomes following neonatal Resuscitation ( $\mathrm{N}=213)$

Before SDA $(\mathrm{N}=126), \quad$ After $\operatorname{SDA}(\mathrm{N}=87), \quad$ P-Value (Fisher' exact
$\mathrm{n}(\mathrm{Wt} . \%)$ $\mathrm{n}(\mathrm{Wt} . \%)$ test)

\section{Newborns Outcome}

$\begin{array}{llll}\text { Stable } & 39(30.95) & 63(72.41 \%) & P(\text { Fisher })=0.000 \\ \text { Unstable(Transferred) } & 78(61.90) & 24(27.59) \\ \text { Dead } & 9(7.14) & 0(0.00)\end{array}$

Abbreviations: Wt.\%: Weighted percent

\section{Pre-Post Differences in Maternal outcomes following PPH Management}

We found a statistically significant association between the SDA intervention and maternal' outcomes following PPH management, 6 months after baseline (Table 4). Among 114 cases of PPH who took part in the study, stable outcome following PPH management was recorded in $77.61 \%$ and $93.62 \%$ at baseline and endline respectively. Transferred (unstable) women were $19.40 \%$ and $6.38 \%$ at baseline and endline respectively. While death attributed to $\mathrm{PPH}$ appeared in $2.99 \%$ women for the baseline group only. No death was recorded in the endline group. There were slight differences in the proportions values of the three categories of maternal' outcomes (stable, transferred, and dead) following PPH management at baseline, and at endline, the Fisher's exact test p-value was 0.048. 
Table 4

Maternal Outcomes following PPH Management ( $\mathrm{N}=114)$

\begin{tabular}{|llll|}
\hline & $\begin{array}{l}\text { Before SDA (N = 67), } \\
\mathrm{n}(\mathrm{Wt} . \%)\end{array}$ & $\begin{array}{l}\text { After SDA(N = 47), } \\
\mathrm{n}(\mathrm{Wt} . \%)\end{array}$ & $\begin{array}{l}\text { P-Value (Fisher' exact } \\
\text { test) }\end{array}$ \\
\hline Maternal outcome & & & $\mathrm{P}($ Fisher $)=0.048$ \\
\hline Stable & $52(77.61)$ & $44(93.62)$ & \\
\hline Unstable(Transferred) $)$ & $13(19.40)$ & $3(6.38)$ & \\
\hline Dead & $2(2.99)$ & $0(0.00)$ & \\
\hline Abbreviations: Wt.\%: Weighted percent & & \\
\hline
\end{tabular}

\section{Discussion}

\section{Main findings}

The current study assessed the effects of the SDA intervention on basic emergency obstetric and newborn care (BEmONC) outcomes for the most frequent complications in Rwanda; post-partum haemorrhage(PPH) and newborn asphyxia and its complications. Apgar scores and PPH progressions were considered in the investigation of newborn and maternal outcomes following neonatal resuscitation and PPH management.

Our study showed that the 6-month long 'SDA intervention' had beneficial effects particularly for supporting nurses and midwives' decisions for neonatal resuscitation and PPH management in both Masaka and Nyamata district hospitals. The use of the SDA was significantly associated with positive maternal and newborns' outcomes following PPH management and neonatal resuscitation, 6 months after baseline. The after intervention groups had a considerably lower occurrence of referrals for both the newborns and the mothers. There were more stable newborn outcomes following neonatal resuscitation and more stable maternal outcomes following PPH management in the after intervention groups compared with the before intervention groups. Also, the few deaths following neonatal resuscitation and PPH management were only recorded in the before intervention groups.

\section{Comparison with other studies}

As was noted in the present study, Lund et al.[30,31] also reported a significant effect of the use of mhealth technologies and the SDA particularly on skilled birth attendants in terms of significantly increased knowledge and skill scores in neonatal resuscitation in Ethiopia[30]. And, a significant reduction in perinatal mortality in Zanzibar[31]. Also, Tamrat et al.[32] in his systematic review which analysed mHealth interventions in maternal and newborn Health Programs and their outcomes around the world reported that the integration of mobile health for prenatal and newborn health services has demonstrated positive outcomes [32]. This could be explained by the fact that mhealth applications have the potentiality to put relevant and reliable healthcare information into the hands of healthcare workers thus helping them in the management of neonatal cases. But also, that health professionals need continuous access to updated healthcare information and clinical guidelines to support their decision making during practices. The same opinion was reported in a similar study done by Grol et al(2003) about the effective implementation of mhealth interventions [33].

Maternal outcome following PPH management was another significant predictor in the present study. Our findings are in agreement with previous literature on this topic. A 2017 systematic review on mHealth interventions in low- 
income countries to address maternal health demonstrated good promise for the use of mHealth interventions in maternal health[34]. This may be because mHealth interventions have been tested in a variety of health services delivery including decision support and clinical management[35-37]. These studies showed promising findings for the mhealth technology in general and for maternal healthcare services in particular. A similar review which identified 51 RCTs, documented that more than half $(n=29)$ of these studies had positive results supporting the effectiveness of mHealth interventions[38]. However, there was still a need for more evidence and higher-quality research in this field. The current study adds to much-needed evidence as to how a mHealth-based clinical decision support tool functioned in a low-resource setting and its linkage with maternal' and neonatal' outcomes.

\section{Limitations}

Though our study documents important patterns of use of a mHealth to inform clinical decisions, the kind and quantity of information accessed for the care of individual patients are undetermined in the current study. Also, nurses and midwives may have consulted other sources rather than the SDA to inform their decision-making during the intervention. Although this limitation is inherent in the design of the study, the current study provides muchneeded insights as to how a mHealth-based clinical decision support tool functioned in a low-resource setting.

\section{Conclusion}

The use of the SDA supported nurses and midwives in the management of PPH and neonatal resuscitation thus improved maternal and neonatal outcomes, six months after baseline. mHealth interventions that focus on the clinical decision support process in obstetric and newborn care may contribute to improved services delivery and should be considered by policymakers in resource-limited settings, like Rwanda. Overall, there is limited evidence on the effects of mHealth interventions on a large scale and further researches are necessary to draw holistic conclusions, particularly for developing countries within the field of maternal and newborn care.

\section{Abbreviations}

BEmONC: Basic Emergency Obstetric and Newborn Care; LMICs: Low- and middle-income countries; mHealth: Mobile Health, SDA: Safe Delivery mhealth Application; PPH: Post-Partum Hemorrhage, NR: Neonatal Resuscitation; SBA: Skilled Birth Attendants, WHO: World Health Organisation; UNICEF: United Nations International Children's Emergency Fund; UNFPA: United Nations Population Fund.

\section{Declarations}

\section{Authors' contributions}

$A N, L I, M N$, and DNC were involved in the conception and design of the study. AN drafted the manuscript. LI, MN, and DNC reviewed the manuscript. All authors read and approved the final manuscript.

\section{Acknowledgments}

We would like to thank the Consortium for Advanced Research and Training (CARTA), The Ministry of Health, Rwanda, and The Maternity Foundation.

\section{Competing interests}

Page $12 / 16$ 
None declared.

\section{Availability of data and materials}

The dataset generated for this study will be made available from the corresponding author on a reasonable request.

\section{Consent for publication}

Not applicable.

\section{Ethics approval and consent to participate}

This study has been approved by the Human Research Ethics Committee of the University of the Witwatersrand (M190258) and the University of Rwanda, College of Medicine and Health Sciences' Institutional Review Board (No.377/CMHS IRB/2018). Permission has been granted by the hospitals.

\section{Funding}

This research was supported by the Consortium for Advanced Research Training in Africa (CARTA). CARTA is jointly led by the African Population and Health Research Center and the University of the Witwatersrand and funded by the Carnegie Corporation of New York (Grant No-B 8606.R02), Sida (Grant No:54100113), the DELTAS Africa Initiative (Grant No: 107768/Z/15/Z) and Deutscher Akademischer Austauschdienst (DAAD). The DELTAS Africa Initiative is an independent funding scheme of the African Academy of Sciences (AAS)'s Alliance for Accelerating Excellence in Science in Africa (AESA) and supported by the New Partnership for Africa's Development Planning and Coordinating Agency (NEPAD Agency) with funding from the Wellcome Trust (UK) and the UK government. The statements made and views expressed are solely the responsibility of the Fellow". The funding body/bodies played no role in the design of the study and data collection process, analysis, interpretation of data, and in writing the manuscript.

\section{References}

1. Payne JD. The State of Standards and Interoperability for mHealth among Low-and Middle-Income Countrieshttp://www.mhealthknowledge.org/sites/default/files/12_state_of_standards_report_2013.pdf (2013, accessed 28 March 2018).

2. Free C, Phillips G, Watson L, et al. The Effectiveness of Mobile-Health Technologies to Improve Health Care Service Delivery Processes: A Systematic Review and Meta-Analysis. PLoS Med, 10. Epub ahead of print 2013. DOI: 10.1371/journal.pmed.1001363.

3. Hall CS, Fottrell E, Wilkinson S, et al. Assessing the impact of mHealth interventions in low- and middle-income countries - what has been shown to work? Epub ahead of print 2014. DOI: 10.3402/gha.v7.25606.

4. Lee SH, Nurmatov UB, Nwaru BI, et al. Effectiveness of mHealth interventions for maternal, newborn and child health in low- and middle-income countries: Systematic review and meta-analysis. J Glob Health; 6. Epub ahead of print 2016. DOI: 10.7189/jogh.06.010401.

5. Wallis L, Blessing $P$, Dalwai $M$, et al. Integrating mHealth at point of care in low- and middle-income settings: The system perspective. Glob Health Action; 10. Epub ahead of print 2017. DOI: 10.1080/16549716.2017.1327686.

6. Tomlinson M, Rotheram-Borus MJ, Swartz L, et al. Scaling Up mHealth: Where Is the Evidence? PLoS Med 2013; 10: e1001382. 
7. Huq NL, Azmi AJ, Quaiyum MA, et al. Toll free mobile communication: overcoming barriers in maternal and neonatal emergencies in Rural Bangladesh. Reprod Health 2014; 11: 1-12.

8. Martinez B, Ixen EC, Hall-Clifford R, et al. mHealth intervention to improve the continuum of maternal and perinatal care in rural Guatemala: a pragmatic, randomized controlled feasibility trial. Reprod Health 2018; 15 : 120.

9. Ilozumba O, Abejirinde IOO, Dieleman M, et al. Targeting strategies of mHealth interventions for maternal health in low and middle-income countries: A systematic review protocol. BMJ Open; 8. Epub ahead of print 2018. DOI: 10.1136/bmjopen-2017-019345.

10. Lee SH, Nurmatov UB, Nwaru BI, et al. Effectiveness of mHealth interventions for maternal, newborn and child health in low-and middle-income countries: Systematic review and meta-analysis. J Glob Health; 6.

11. Mburu S, Oboko R. A model for predicting utilization of mHealth interventions in low-resource settings: case of maternal and newborn care in Kenya. BMC Med Inform Decis Mak, 18. Epub ahead of print 2018. DOI: 10.1186/s12911-018-0649-z.

12. Amoakoh HB, Klipstein-Grobusch K, Amoakoh-Coleman M, et al. The effect of a clinical decision-making mHealth support system on maternal and neonatal mortality and morbidity in Ghana: study protocol for a cluster randomized controlled trial. Trials 2017; 18: 157.

13. Nyamtema A, Mwakatundu N, Dominico S, et al. Introducing eHealth strategies to enhance maternal and perinatal health care in rural Tanzania. Matern Heal Neonatol Perinatol 2017; 3: 3.

14. Free C, Phillips G, Watson L, et al. The Effectiveness of Mobile-Health Technologies to Improve Health Care Service Delivery Processes: A Systematic Review and Meta-Analysis. PLoS Med 2013; 10: e1001363.

15. Philbrick WC. mHealth and MNCH: State of the Evidence. Trends, Gaps, Stakeholder Needs, and Opportunities For Future Research on the Use of Mobile Technology to Improve Maternal, Newborn, and Child Health. mHealth Alliancehttp://www.healthenabled.org/resources/pmnch_mhealth_evidence.pdf (2013, accessed 26 May 2017).

16. Nurmatov UB, Lee SH, Nwaru BI, et al. The effectiveness of mHealth interventions for maternal, newborn and child health in low- and middle-income countries: Protocol for a systematic review and meta-analysis. $J$ Glob Health 2014; 4: 010407.

17. Hagar $\mathrm{C}$, Kartzinel $\mathrm{H}$. Healthcare information for all by 2015: preliminary findings and future direction. Inf Dev 2016; 32: 354-361.

18. Thomsen CF, Barrie AMF, Boas IM, et al. Health workers' experiences with the Safe Delivery App in West Wollega Zone, Ethiopia: a qualitative study. Reprod Health 2019; 16: 50.

19. Granja C, Janssen W, Johansen MA. Factors determining the success and failure of ehealth interventions: Systematic review of the literature. Journal of Medical Internet Research; 20. Epub ahead of print 2018. DOI: $10.2196 / 10235$.

20. Labrique $A B$, Vasudevan $L$, Kochi $E$, et al. mHealth innovations as health system strengthening tools: 12 common applications and a visual frameworkhttp://southasiainstitute.harvard.edu/website/wpcontent/uploads/2014/08/MHEALTHINNOVATIONS.pdf (2015, accessed 20 March 2017).

21. Hagar C, Kartzinel H. Healthcare Information For All By 2015. Inf Dev 2016; 32: 354-361.

22. Maternity Foundation $M$ for mothers. What is the SDA app - Maternity Foundationhttps://www.maternity.dk/about-the-app/what-is-the-app/ (2014, accessed 26 July 2018).

23. Rwanda NI of S of, Planning/Rwanda M of F and E, Health/Rwanda M of, et al. Rwanda Demographic and Health Survey 2014-15https://dhsprogram.com/publications/publication-fr316-dhs-final-reports.cfm (2016, 
accessed 14 November 2019).

24. Ngabireyimana E, Mutaganzwa C, Kirk CM, et al. A retrospective review of the Pediatric Development Clinic implementation: a model to improve medical, nutritional and developmental outcomes of at-risk under-five children in rural Rwanda. Matern Heal Neonatol Perinatol 2017; 3: 13.

25. MOH RHP. REPUBLIC OF

RWANDAhttp://www.moh.gov.rw/fileadmin/templates/policies/Health_Sector_Policy__19th_January_2015.pdf (2015, accessed 7 August 2017).

26. Wilmot E, Yotebieng M, Norris A, et al. Missed Opportunities in Neonatal Deaths in Rwanda: Applying the Three Delays Model in a Cross-Sectional Analysis of Neonatal Death. Matern Child Health J 2017; 21: 1121-1129.

27. Puri R, Rulisa S, Joharifard S, et al. Knowledge, attitudes, and practices in safe motherhood care among obstetric providers in Bugesera, Rwanda. Int J Gynecol Obstet 2012; 116: 124-127.

28. Namusoke $\mathrm{H}$, Nannyonga MM, Ssebunya R, et al. Incidence and short term outcomes of neonates with hypoxic ischemic encephalopathy in a Peri Urban teaching hospital, Uganda: a prospective cohort study. Matern Heal Neonatol Perinatol 2018; 4: 6.

29. Website MF. Our Work - Maternity Foundationhttps://www.maternity.dk/our-work/ (accessed 23 July 2020).

30. Lund S, Boas IM, Bedesa T, et al. Association Between the Safe Delivery App and Quality of Care and Perinatal Survival in Ethiopia: A Randomized Clinical Trial. JAMA Pediatr 2016; 170: 765-771.

31. Lund S, Rasch V, Hemed M, et al. Mobile phone intervention reduces perinatal mortality in zanzibar: secondary outcomes of a cluster randomized controlled trial. JMIR mHealth uHealth 2014; 2: e15.

32. Tamrat T, Kachnowski S. Special delivery: An analysis of mhealth in maternal and newborn health programs and their outcomes around the world. Matern Child Health J 2012; 16: 1092-1101.

33. Grol R, Grimshaw J. From best evidence to best practice: effective implementation of change in patients' care. Lancet 2003; 362: 1225-1230.

34. Colaci D, Chaudhri S, Vasan A. mHealth Interventions in Low-Income Countries to Address Maternal Health: A Systematic Review. Ann Glob Heal 2017; 82: 922.

35. McNabb M, Chukwu E, Ojo O, et al. Assessment of the quality of antenatal care services provided by health workers using a mobile phone decision support application in northern Nigeria: A pre/post-intervention study. PLoS One; 10. Epub ahead of print 2015. DOI: 10.1371/journal.pone.0123940.

36. Ngoc NTN, Bracken H, Blum J, et al. Acceptability and feasibility of phone follow-up after early medical abortion in Vietnam: a randomized controlled trial. Obstet Gynecol 2014; 123: 88-95.

37. Chib A. The Aceh Besar midwives with mobile phones project: Design and evaluation perspectives using the information and communication technologies for healthcare development model. J Comput Commun 2010; 15 : $500-525$.

38. Chen $\mathrm{H}$, Chai Y, Dong L, et al. Effectiveness and Appropriateness of mHealth Interventions for Maternal and Child Health: Systematic Review. JMIR mHealth uHealth 2018; 6: e7.

\section{Figures}




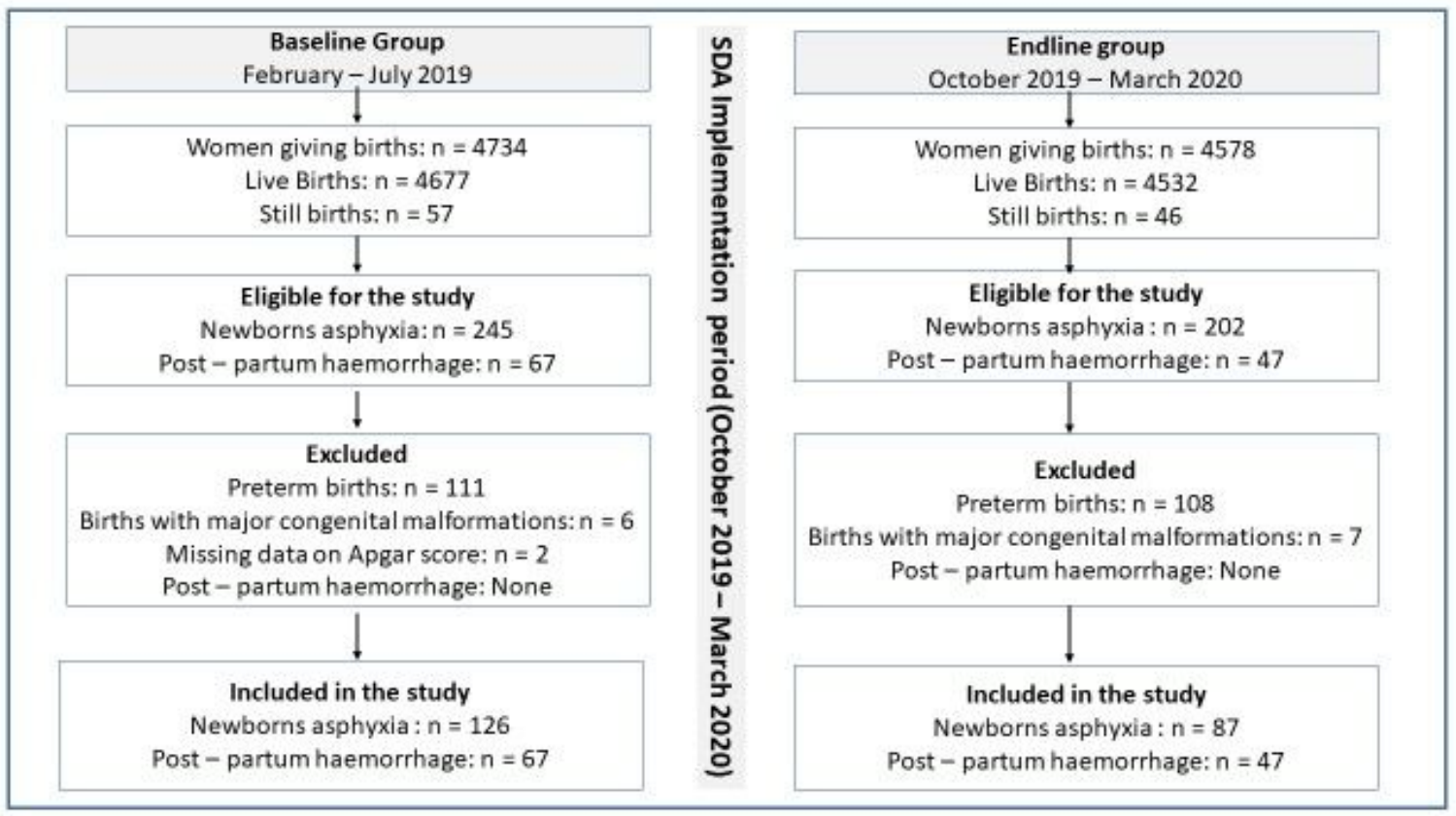

Figure 1

Records review flow chart 